\title{
Wer hat Angst vor der Biologie?
}

Anlage oder Umwelt? Viele Jahrzehnte lang beherrschte diese Frage in immer wieder wechselnden Konjunkturen die Suche nach den Determinanten strafbaren und abweichenden Verhaltens. Diese Zeiten sind nun vorbei. Die Tagung „Gefährliche Menschenbilder“ bringt Kriminologen und Neurologen zusammen.

Dass die soziale Situation des Menschen mit seinen biologischen und psychischen Merkmalen und Zuständen in einer permanenten Wechselbeziehung steht, kann heute als Konsens zwischen Neurowissenschaftlern, Psychologen und kritischen Kriminologen gelten. Das wurde schnell deutlich, als sich über 100 Wissenschaftler und Wissenschaftlerinnen im Bielefelder Zentrum für interdisziplinäre Forschung unter der Überschrift „Gefährliche Menschenbilder“ zu Diskussionen über „Bio-Wissenschaften, Gesellschaft und Kriminalität“ zusammengefunden hatten. Und doch: Viel erreicht war mit dieser Einigkeit noch nicht. Wenn auch das dichotome Denken über die individuellen Hintergründe von kriminalisierbaren Verhaltensweisen der Vergangenheit angehört, so erweisen sich die Fragen nach der Bewertung und den gesellschaftlichen Folgen jüngerer neurobiologischer Erkenntnisse nun sogar als noch schwieriger als die alten Streitfragen. Welchen Beitrag können Hirnforscher und Biologen zum Verständnis von Kriminalität liefern? Ist die Prognose delinquenten Verhaltens überhaupt möglich? Welches Konzept von (delinquenten) Menschen kündigt sich in einer Gesellschaft an, die sich verstärkt mit der Hilfe von Computertomographen und GenAnalysen auf die Suche nach dem Wesen des „Menschseins“ macht?

Seit einigen Jahren schon offerieren die so genannten „Lebenswissenschaften, “ vor allem die neurobiologische Hirnforschung, neue Perspektiven auf den Menschen und fordern damit die gesamten Kriminalwissenschaften heraus. Die uralten Debatten über die menschliche Willensfreiheit als Grundlage des strafrechtlichen Schuldvorwurfes sind neu entflammt, zwischen Psychoanalyse und Neurobiologie entstehen neue Debatten (Ansermet/Magistretti 2005) und in die Kriminologie sind die Biowissenschaften plötzlich und unerwartet wieder zurückgekehrt (Strasser 2005). Dabei ist es vor allem die reduktionistische Vorstellung, den „Sitz des Bösen “1 in einem menschlichen Gehirn verorten und Delinquenz anhand dieser Daten prognostizieren zu können, die zu einem veränderten Umgang nicht nur mit Kriminalität, sondern mit allen potentiellen Kriminellen - also mit sämtlichen Bürgern - führen könnte. Für die Veranstalter $^{2}$ der Bielefelder Tagung drängte sich daher die Frage auf, ob mit den Methoden der Hirnforschung allein elektronische „Bilder gefährlicher Menschen“ geliefert werden, oder ob hier zugleich auch „gefährliche Menschenbilder“ in einer Gesellschaft entstehen. Diese Gefahr machte Mitveranstalter Lorenz Böllinger, Psychologe und Strafrechtler in Bremen, schon zu Beginn der Tagung deutlich und verwies auf den dringenden Bedarf nach einem interdisziplinären Austausch von Erkenntnissen und Positionen innerhalb dieses Themenfeldes. Denn einen Austausch von Neuro-Wissenschaftlern, Psychologen, Sozial-, Kulturwissenschaftlern und Kriminologen über die mögliche Relevanz der jüngsten Forschung und ihrer Thematisierung für den gesellschaftlichen Umgang mit Kriminalität gab es bisher kaum. Zwar wurde bereits auf einer Reihe von Tagungen die Bedeutung der lebenswissenschaftlichen Forschung für einzelne Disziplinen thematisiert, doch die gesellschaftlichen Funktionen der
Lebenswissenschaften in kriminologischer und kriminalpolitischer Hinsicht blieben bisher weitgehend im Dunkeln.

Vor diesem Hintergrund war es nicht überraschend, dass schon das Auftaktreferat des Neurobiologen Hans Joachim Markowitsch (Bielefeld) zu kontroversen Diskussionen führte. Markowitsch erläuterte anhand von Beispielen aus der Evolutionsforschung und dem Tierreich, wie sehr das menschliche Verhalten durch ein Wechselspiel von genetischer Grundausstattung und Umweltfaktoren determiniert sei. Deutlich wurde das - auch aus Sicht der Neurobiologie - interaktionistische Verhältnis von Konstitution und Kontext durch Markowitschs Hinweis, dass zahlreiche genetische Strukturen des Menschen erst durch Impulse aus der Umwelt jeweils „,an- oder abgeschaltet“ werden. Diese sozialen Faktoren würde sich nach seiner Ansicht aber in hirnorganischen Veränderungen manifestieren, die das Verhalten des Individuums in bestimmten Grenzen vorhersagbar werden ließen. Auf erheblichen Widerspruch von Kriminologen stieß der Neurowissenschaftler mit seiner These, Kriminalität sei daher grundsätzlich als eine Ausprägung (hirn-)kranken Verhaltens $\mathrm{zu}$ verstehen und zwischen forensisch und nicht-forensisch Straffälligen bestünden nur graduelle Unterschiede. Diese Pathologisierung der Abweichung wurde von einigen Konferenzgästen als eine Sichtweise kritisiert, die keine Rücksicht auf die soziale Konstruktion des Phänomens Kriminalität nimmt. „Völlig unklar“ bliebe, so bemerkte etwa Helge Peters (Oldenburg), welcher Begriff von Kriminalität dieser Verbindung von Verbrechen und Hirnstruktur zugrunde liegt. In eine ähnliche Richtung argumentierte Karl-Ludwig Kunz (Bern), der in seinem Referat die Biologie als Erklärungswissenschaft für Kriminalität entschieden ablehnte, weil sie die Gesellschaft als Aushandlungsinstanz der Inhalte von „Kriminalität“ ignorieren würde.

Welche Auswirkungen der Fokus auf die Gene bereits heute auf das „Menschsein“ in der Gesellschaft hat, machte Barbara Prainsack (London) am Beispiel US-amerikanischer Biotechnologie-Firmen deutlich. Diese Unternehmen bieten nunmehr auch in Europa für jedermann eine komplette DNA-Analyse an, deren Ergebnisse nach Wunsch mit Familienmitgliedern und Freunden auf einem InternetPortal verglichen werden kann. „Wir werden zu Managern unseres eigenen Gesundheitsprojektes“ sagte sie über die neo-liberale Funktionalisierung individueller genetischer Informationen, die innerhalb gesellschaftlicher Beziehungen allerdings auch leicht zum sozialen Sprengstoff werden können. Zugleich zeigte Prainsack anhand einer Interview-Studie mit Strafgefangenen auf, wie sehr alte Kategorien wie die der „Verbrecher“ oder der „Gefährlichen“ mit Hilfe der DNA-Technologie verfestigt und - in der Terminologie Foucaults - in den Körper eingeschrieben werden. Besonders an den neuen Verfahren sei, dass die DNA-Technik mit dem Anspruch auf Fehlerfreiheit - und damit als ein absoluter Zugang zur „Wahrheit“ gedacht werde. Das Machtgefälle zwischen den Anwendern der DNA-Technik an der Spitze einer Gesellschaft und den Objekten ihrer Anwendung an den sozialen Rändern werde dadurch immens verstärkt.

Die seit mehr als einhundert Jahren immer wieder kehrenden biowissenschaftlichen Erklärungsmuster in der Kriminologie veranschaulichte Richard Wetzell (Washington) eindrucksvoll. Seit den Schriften des italienischen Mediziners Lombroso fanden sich auch in der deutschen Kriminologie zwischen 1890 und 1945 in erster Linie 
von Medizinern vertretene Thesen, die einen engen Zusammenhang zwischen geistigen Abnormitäten und Delinquenz suggerierten, berichtete Wetzell. Obwohl der kriminologische Mainstream die Rolle der Umweltfaktoren stets im Auge behielt und selbst die Mehrheit der Ärzte nie an die Existenz eines „geborenen Verbrechers“ im Sinne Lombrosos glaubte, behielten biologistische Ansätze durch das verstärkte Interesse an der Eugenik während der Weimarer Republik und schließlich durch die biologisch-rassistische Ausrottungspolitik durch die Nationalsozialisten eine erhebliche Anziehungskraft. „Man brauchte gar keine völlig gleichgeschaltete Wissenschaft während der NS-Zeit“, erinnerte der Historiker, die Kontinuität der „ganz normalen“ Wissenschaften reichte den Machthabern für die Verfolgung ihrer eugenischen Strategie aus. Wetzell stellte heraus, dass die von biologisch orientierten Kriminologen vertretene „Unverbesserlichkeit“ konkreter Straftäter zumeist überhaupt nicht auf einer behaupteten primären Bedeutung von Anlagefaktoren beruhte. Hintergrund dieser Prognosen sei vielmehr die pessimistische Einstellung gewesen, die kriminogenen Umweltfaktoren ließen sich ohnehin nicht ändern - wodurch ein biokriminologischer Reduktionismus als pragmatischste Lösung erscheinen musste.

Das dichotome Verständnis von Sozial- und Lebenswissenschaften wollten Reinhard Kreissl (Wien) und Heinz Steinert (Frankfurt) durchbrechen, die darauf hinwiesen, dass die biologische Grundlage des menschlichen - und damit des gesellschaftlichen - Lebens selbstverständlich und unbestreitbar ist. Erklärungsbedürftig sei vielmehr, warum die Hirnforschung heute überhaupt vorgeben könne, gesellschaftstheoretisches Neuland zu betreten. Die falsche Dichotomie zwischen einem soziologischen und einem biologischen Determinismus müsse überwunden werden, meinten Steinert und Kreissl, da sich soziale Ungleichheit nicht nur in den gesellschaftlichen Bedingungen, sondern auch in den Körpern, Hormonen und Genen niederschlage. Sie schlugen vor, die zweifache Prägung der Menschen „durch nature und nurture“ parallel zu betrachten. Eine kritische Theorie sozialer Ausschließung hätte die Formung der Organismen ebenso wie die der sozialen Akteure zu thematisieren.

Pragmatische Konsequenzen aus der modernen Empirie zog Jens Asendorpf (Berlin), der ein eigenes Entwicklungsmodell für deviantes Verhalten präsentierte. Der Psychologe verwies darauf, dass in der jüngeren Forschung in Bezug auf antisoziales Verhalten mehrfach eine Genom-Umwelt-Interaktion im Sinne einer gegenseitigen Potenzierung biologischer und sozialer Faktoren nachgewiesen worden sei. Und die Prognose delinquenter und aggressiver Verhaltensweisen, so Asendorpf, sei mit der geeigneten Methodik durchaus möglich: Längsschnittstudien, in denen die Erfahrungen von Kindergartenbetreuern in Interviews fruchtbar gemacht worden sind, hätten eine signifikant sichere Prognose darüber ermöglicht, ob sich die Probanden später im Alter zwischen 18 und 23 Jahren antisozial verhalten würden. Frühzeitige Intervention bei derart identifizierten Risikogruppen könne somit eine spätere Delinquenz zumindest unwahrscheinlicher machen. Die Thematisierung dieser Verbindungen zwischen Hirnforschung, gesamtgesellschaftlicher Präventionseuphorie und dem Umgang mit Abweichung auch unterhalb der Strafbarkeitsschwelle kam in den Diskussionen oft etwas zu kurz, liegt doch gerade darin ein Potential für die Verbreitung „gefährlicher Menschenbilder.“

Was aber bleibt insgesamt an Erkenntnisfortschritt aus den interdisziplinären Debatten für die Kriminalwissenschaften? „So gefährlich ist das Menschenbild der Hirnforscher nun auch wieder nicht, " resümierte Heinz Steinert mit Blick auf die nach seinem Eindruck eher begrenzte Aussagekraft der bisherigen neurologischen Forschung. Zweifel an der methodologischen Belastbarkeit der empirischen Ergebnisse von Hirnforschung und Gentechnik wurden vielfach formuliert. So hat für Richard Wetzell die Neurobiologie bislang lediglich aufgezeigt, dass zwischen Umweltfaktoren und neuronalen Netzen eine Wechselwirkung besteht und bestimmte Hirnregionen bei bestimmten Tätigkeiten aktiver sind als andere. Diese Erkenntnisse, so Wetzell, stellten „ein eher lächerliches Ergebnis dar für eine Forschung, in die jährlich Millionenbeträge investiert werden.“

Doch die Mehrheit der Kriminologen stand den Lebenswissenschaften mit einer Mischung aus Skepsis und Sorge gegenüber. Drastisch beschrieb Heinz Giehring (Hamburg) seine Befürchtungen, indem er in der gesellschaftlichen und kriminalpolitischen Euphorie über die neuro-biologischen Methoden ein „Gefahrenpotential für Prinzipien, die sich der Rechtsstaat in Jahrhunderten erarbeitet hat" erahnte, da die Grenzen zwischen Gefahrenabwehr, Gefahrenvorsorge und Strafe verschwinden. Skeptisch äußerte sich auch Hans-Jürgen Kerner (Tübingen), der durch Veränderungen von Wahrnehmungsund Funktionalisierungsprozessen durchaus eine von den lebenswissenschaftlichen Studien ausgehende Gefahr in Bezug auf ihre gesellschaftlichen Folgewirkungen sah. Fatal wäre es, meinte der Tübinger Kriminologe, wenn die Lebenswissenschaften für sich eine „unangemessene Deutungshoheit“ über kriminologische Fragen beanspruchen oder erreichen würden. Zugleich zeigte sich Kerner aber optimistisch, dass die neuro-biologischen Erkenntnisse gute Argumente für eine kriminalpolitische Strategie nach dem Motto „Im Zweifel weniger Strafe“ liefern können. Karl F. Schumann (Bremen) gab sich ähnlich zuversichtlich: Die Strafrechtswissenschaft wird auch die Neurowissenschaften erfolgreich abwehren und absorbieren können und das Schuldstrafrecht verteidigen, prognostizierte er. Für die Zukunft forderte er von der Kriminologie größere Forschungsanstrengungen im Prognosebereich, insbesondere hinsichtlich der Entstehungsbedingungen von Gutachten, die fälschlich eine negative Legalprognose für die Probanden stellen. Leider nur wenige Diskutanten beschäftigten sich mit den Hintergründen der neuen Konjunktur der Lebenswissenschaften. Einen Ansatz dafür lieferte Rüdiger Lautmann (Bremen), indem er die Welle der biologistischen Erklärungsmuster für Kriminalität als „ein Produkt zunehmender Kontingenz" bezeichnete. Die Unsicherheit der Menschen in allen Lebensbereichen, insbesondere bei der sozialen, wirtschaftlichen und beruflichen Integration, führe zu der außergewöhnlichen Attraktivität scheinbar sicherer und objektiver Deutungsansätze.

In wesentlichen Bereichen, das machte die Tagung deutlich, sind Kommunikationsstörungen in Folge von grundlegend unterschiedlichen Denkweisen zwischen Natur- und Sozialwissenschaftlern derzeit vorherrschend: Während einige kritische Kriminologen schon die Validität und Relevanz der neurowissenschaftlichen Erkenntnisse negieren, fehlt es manchen Naturwissenschaftlern und Psychologen an jeglicher Reflektion über die gesellschaftlichen Folgen ihrer Thesen und die Legitimation für ihre therapeutischen Forderungen und Vorschläge. Ein umfangreicher Austausch zwischen den Disziplinen, für den die dreitägige Konferenz nur einen ersten Anreiz bieten konnte, erscheint dringend erforderlich. Und von allen Seiten - von Psychologen und Juristen ebenso wie von Historikern und Kriminologen - wurde in Bielefeld vielfach der Wille zu einer positiven Zusammenarbeit anstatt eines antagonistischen Wettstreits der Disziplinen beschworen. In dieser Richtung beschrieb auch Lorenz Böllinger die Aufgabe für die Wissenschaftler der kommenden Jahre: Es wäre viel erreicht, meinte der Kriminologe, wenn in naher Zukunft 
zwischen beiden Wissenschaftsbereichen eine Einigung auf eine Art Forschungsmatrix für das Verständnis kriminologisch relevanter Handlungen geschaffen werden könnte. Ziel dabei müsse es schlicht sein, menschliche Handlungen besser zu verstehen. Doch vor einer derartigen Kooperation von Sozial- und Lebenswissenschaftlern scheint derzeit noch ein steiniger Weg zu liegen.

Dr. Michael Jasch ist wissenschaftlicher Mitarbeiter am Institut für Kriminalwissenschaften der Goethe-Universität Frankfurt am Main. Kontakt:Jasch@jur.uni-frankfurt.de

\section{Fußnoten:}

1 So der suggestive Titel einer Fernsehdokumentation über die Suche nach Kriminalitätsursachen in den Gehirnen verurteilter Gewalttätern, die erstmals 2006 von den Fernsehprogrammen 3sat und Phoenix ausgestrahlt wurde.

2 Wissenschaftliche Veranstalter der Tagung waren die Vorstandsmitglieder der Gesellschaft für interdisziplinäre wissenschaftliche Kriminologie (GiwK).

\section{Literatur:}

Ansermet, Francois; Magistretti, Peter (2005): Die Individualität des Gehirns: Neurobiologie und Psychoanalyse, Frankfurt a.M.

Strasser, Peter (2005): Die Rückkehr der Bio-Wissenschaften in der Kriminologie, in: Pilgram, A.; Prittwitz, C. (Hrsg.): Kriminologie. Akteurin und Kritikerin gesellschaftlicher Entwicklungen (Jahrbuch für Rechts- und Kriminalsoziologie), Baden-Baden.

\section{Bericht über das Wiener Forum gegen Menschen- handel (Tagung vom 13.-15.02.2008)}

Ina Hunecke

\begin{abstract}
Menschenhandel ist ein globales Problem. Die Erkenntnis, dass seine Bekämpfung nur durch Zusammenwirken vieler Staaten Erfolg versprechend ist, veranlasste die UN.GIFT ${ }^{1}$ (Global Initiative to fight human trafficking) die erste große UNO-Konferenz mit ca. 1.600 Teilnehmern aus mehr als 128 Staaten vom 13. bis 15. Februar 2008 in Wien einzuberufen.

Gastgeberin war die Nationalratspräsidentin Barbara Prammer. Sie sah die Hauptaufgabe der Tagung darin, nicht nur ein Netzwerk zu knüpfen, sondern einen weiteren Meilenstein im Kampf gegen den Menschenhandel zu setzen. Diesem Wunsch gab sie in ihrer Begrüßungsrede deutlich Ausdruck, als sie sagte: „Ziel müsse es sein, die entsprechenden internationalen Vereinbarungen - die UN-Konvention gegen internationale Verbrechen, das Zusatzprotokoll zur Verhinderung, Verfolgung und Bestrafung von Menschenhandel sowie die diesbezügliche Konvention des Europarates und die Brüsseler Deklaration - zu implementieren und durch konkrete Maßnahmen mit Leben zu füllen.“
\end{abstract}

Der Exekutivdirektor des UN-Büros für Drogen- und Verbrechensbekämpfung Antonio Maria Costa verglich den Menschenhandel mit der Sklaverei und erinnerte an den britischen Unterhaus-Abgeordneten William Wilburforce, der vor 200 Jahren maßgeblich zum Verbot des Sklavenhandels beigetragen hatte. Er rief die Parlamentarier auf, sich daran ein Beispiel zu nehmen und auf nationaler Ebene die notwendigen legislativen Maßnahmen zu treffen und das machtvolle Instrument des UN-Protokolls zur Bekämpfung des Menschenhandels umzusetzen.

Weitere Gastredner waren unter anderen H.E. Suzanne Mubarak, Julia Ormond, Ruslana Lyzhychko, Ricky Martin sowie viele andere Persönlichkeiten aus Politik und Gesellschaft.

Die Themen der Diskussionsforen waren so vielfältig wie die Redner und Besucher ebendieser. Es ging um Prävention, die vielfältigen Gründe für Menschenhandel, die Rolle der Medien, die Frau als Menschenhändler, den Bali Prozess als Model für regionale Kooperation im Kampf gegen Menschenhandel, die Rolle der Jugend, der
Kirchen sowie Glaubengemeinschaften. Es ging um Korruption, um Strategien auch bildungsarme Länder und Schichten zu erreichen sowie immer wieder um die zentrale Frage „Wie kann wer mit wem zusammenarbeiten, um die aktuelle Situation zu verbessern.“

Über die Diskussionsforen hinaus bot die Veranstaltung eine Fülle von Möglichkeiten sich mit dem Thema Menschenhandel sowie Vermeidungs- und Aufklärungsstrategien auseinanderzusetzen.

Eine der kreativen Aktionen startete bereits im Vorfeld des Forums. Die UN.GIFT rief einen weltweiten Comic-Wettbewerb aus, mit dem vor allem Jugendliche erreicht und für das Thema sensibilisiert werden sollten. Die Gewinnercomics des Wettbewerbs waren auf Bannern während des Forums zu sehen und werden in Zukunft von der UN.GIFT genutzt werden, um gerade auch junge Menschen in der ganzen Welt, unabhängig von Sprache und Intellekt zu erreichen und auf das Problem aufmerksam zu machen.

Darüber hinaus wird ein virtuelles Netz erstellt, um weiter miteinander in Kontakt zu bleiben und Aktionen sowie Veranstaltungen planen zu können.

Des Weiteren wurden auch Modellprojekte von Privaten und Unternehmen vorgestellt. Hierfür exemplarisch ist YCI. In diesem Projekt, welches 1995 in Bangkok startete, wird jungen Menschen ein halbes Jahr lang die Möglichkeit gegeben, sich in einem Hotel sowohl theoretisch als auch praktisch ausbilden zu lassen. Die jungen Leute erhalten so eine Perspektive. Wer Arbeit hat, wird sich nicht einem Menschenhändler ausliefern, war eine der gründenden Ideen zu diesem Projekt. Inzwischen gibt es solche „Ausbildungshotels“ auf der ganzen Welt und mehr als 1.600 junge Menschen erhielten so bereits eine Chance auf ein besseres Leben.

Neben den Diskussionsforen setzten sich die Teilnehmer auch cineastisch mit dem Thema auseinander. Gezeigt wurden 19 Filme, darunter auch der auf dem Filmfest in München preisgekrönte Film „Trade“ oder „Blood Diamond“. 